\title{
Study on Driving Dangerous Area in Road Curved Section Based on Vehicle Track Characteristics
}

\author{
REN Yuan-yuan, LI Xian-sheng, REN You* \\ Traffic College of Jilin University \\ Chuangchun, 130022, China \\ Rachel GUAN \\ Minnesota Department of Transportation \\ 395 John Ireland Blvd. St. Paul, 55155, America \\ GUO Wei-wei \\ School of Mechanical Engineering, Beijing Institute of Technology \\ Beijing 100081, China \\ Received 10 March 2011; accepted 25 November 2011
}

\begin{abstract}
Plenty of experiments and data analysis of vehicle track type in road curved section show that the deviation and the crossing characteristics of vehicle track paths are directly related to the driving stability and security. In this paper, the concept of driving dangerous area in curved section was proposed based on the vehicle track characteristics according to driving behavior decision making and accident probability. Taking the observation data of passenger cars as the representative, the vehicle track paths deviation regularity per measurement direction were analyzed by SPSS under the condition of different radius and different road cross section. The distribution probability of different track type and the accident probability calculation methods and results between opposite direction vehicles were given based on the track characteristics.
\end{abstract}

Keywords: Vehicle Track Type; Track Deviation; SPSS Analysis, Accident probability; Driving Decision Making, Driving Dangerous Area.

\section{Introduction}

As shown by substantial accident data, horizontal curved section is closely linked to road safety. The accident severity is more severe compared with straight sections, about $25 \% \sim 30 \%$ of fatal accidents occurred on curved sections. Research on road safety at home and abroad shows that: more than $80 \%$ of traffic accidents are directly or indirectly related with drivers because of the traffic behavior mutation (including driving speed and track) ${ }^{1-2}$.

At present, relevant researches have been carried out in reaction to the problems mentioned above. Some scholars have made analysis of the rule and impact of traffic accidents happened on curved sections ${ }^{3}$. And some scholars also studied the relationship between vehicle track and road safety of small radius curved sections, e.g. I. L. Thomas ${ }^{4}$, SUN Xiao-duan ${ }^{5}$, Peter Spacek ${ }^{6-7}$, E. Donges ${ }^{8}$, etc.. However, no relations and rules are defined between the changes in human-vehicle driving status and the danger of traffic safety. And rarely study on safety of road from vehicle track ${ }^{9-13}$.

Therefore, this paper deeply studies the vehicle track mutability characteristics, and the distribution probability of different track type, the accident probability calculation method and result between

\footnotetext{
*Corresponding author: REN You(1967-), Associate Professor, renyou@jlu.edu.cn.
} 
opposite direction vehicles were given based on the track characteristics.

At last, the different driving dangerous areas of curved section and its characteristics were given combines the risk of traffic accident. The research thinking and conclusions have greatly theoretical and applicable value in reflecting the relationship between traffic behavior and traffic accident more comprehensively and improving the road curved section design and the roadside safety design.

\section{Definition of Driving Dangerous Area in Curved Section}

For simplification purpose, involved key objects in this paper were defined as follows:

(1) Curved section: mainly refer to curved sections of two-way two-lane road without physical separation in the middle, namely, mainly curved sections of roads of secondary level or below.

(2) Driving direction: the direction driving along the outer side of curved section is defined as up direction, while that driving along the inner side of curved section is defined as down direction.

(3) Vehicle track: when the vehicle run into the curved section, the route passed by the left front wheel is defined as vehicle track.

In terms of driving process, the formation process of traffic accident is that, the drivers constantly search and perceive road traffic information in the course of driving, and adjust the running speed and track behavior accordingly. The traffic accident would be happen once no reaction or adjustment in time. And there must be a corresponding dangerous space or dangerous state of a road section which is the driving dangerous area upon the occurrence of concentrated and significant risk avoidance motive and behavior.

Therefore, for convenience of experimental observation and application in safety analysis of curved sections, this paper defined the space-time sections corresponding to the specific period and road section with most dramatic changes in human-vehicle operation status to be dangerous driving area in curved sections.

\section{Experimental Design and Data Analysis}

\subsection{Selection of experimental curved section}

The selected curved section is two-way two-lane road of secondary level, with basically the same corner, and the radius is respectively $110 \mathrm{~m}, 260 \mathrm{~m}$ and $310 \mathrm{~m}$. This road is $9 \mathrm{~m}$ in width, and in a sound condition. Traffic engineering facilities provided for this road is complete.

\subsection{Division of crucial sections of curved section}

For the purpose to obtain the vehicle track characters, the most important observation point is the middle point of the curve, the transitional point from straight section to curved section, and the transitional point from curved section to straight section. According to the road geometry features, there are three points can meet the requirement very well, which respectively is QZ point, $\mathrm{HY}$ point and $\mathrm{YH}$ point. In order to obtain a smooth vehicle track path, another two points between $\mathrm{HY}$ and QZ, QZ and YH also are important. So, at least 5 observation points will be needed in one curved section. To be frank, the more the number of the observation points, the better. But for the limitation of fund and personnel, the curved section in this paper is only divided into five crucial sections, namely, ZH, HY, QZ, $\mathrm{YH}$ and $\mathrm{HZ}$ as reference points in analysis of vehicle track mutability.

\subsection{Selection of vehicle type}

In order to facilitate observing, vehicles in this paper is divided into four types according to their size, which respectively is the large passenger car, the large truck, the small passenger car, the small truck. It is found that the proportion of passenger cars and trucks is $5: 1$, and the number of other three vehicle types is too few to be analyzed, so considering meeting the requirement of vehicle sample size, this paper only collect and analyze the track data of small passenger cars.

\subsection{Data analysis}

The measurements took place during daytime outside, and the sample amount to 1200 vehicles in both direction of three curved sections with different radius in the view of the limitation of two-lane roads of secondary level or below. Because limitation of only 5 
detecting points, in order to get the whole driving track path in curved section, cubic spline interpolation is adopted to fit the 5 detecting data, at last, the whole vehicle track path in curved section will be calculated.

\section{Analysis on vehicle track type and track path mutability characteristics in curved section}

Divers generally use the road center line to guide the sight driving on curved section. According to plenty of experimental data analysis, vehicle track paths coming from up direction deviate inwardly obviously driving into the sharp curved section in the cases without detecting vehicles come from opposite direction, especially in the section driving in or out. And the vehicle track paths from down direction always along the road center line, but an obviously outward deviation when driving out curved section. Thus, this paper take passenger cars for instance, analyze the vehicle track type and track paths mutability characteristics in curved section.

\subsection{Vehicle track type in curved section}

One of the main purposes of this investigation was to define suitable track types that could be observed. According to the observation data and on the basis of test measurements, the following six track types were defined in this paper ${ }^{7}$, see Fig. 1

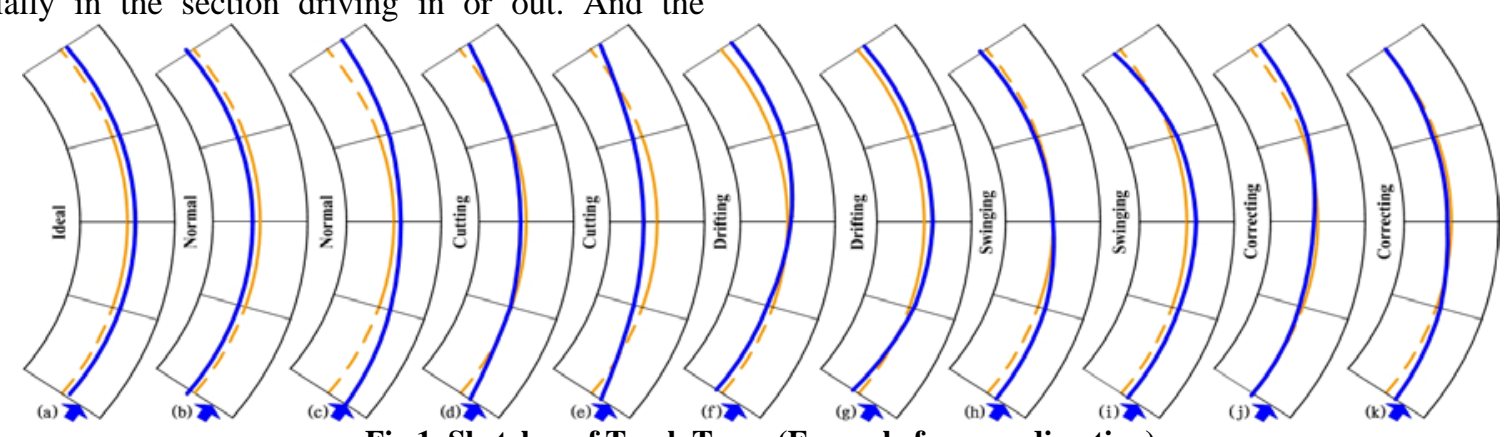

Fig.1 Sketches of Track Types (Example for a up direction)

Table 1 Distribution Probability of Track Types (\%)_ in Investigated Curves

\begin{tabular}{c|c|c|c|c|c|c|c|c|c|c|c}
\hline & (a) & (b) & (c) & (d) & (e) & (f) & (g) & (h) & (i) & $(\mathrm{j})$ & $(\mathrm{j})$ \\
\hline $\mathrm{P}$ & 0.01 & 0.10 & 0.11 & 0.04 & 0.21 & 0.12 & 0.05 & 0.07 & 0.09 & 0.04 & 0.16 \\
\hline
\end{tabular}

(i)Ideal behavior: This is corresponds to a symmetrical track path formed by the vehicle left front wheel within a narrow area along the center of the road. Such an idealized track path is assumed in the design standards.

(ii)Normal behavior: This is likewise indicates a symmetrical track path formed by vehicle left front wheel along the center of road, but within a somewhat broader area than with the ideal behavior and with slight cutting to the road center line.

(iii)Cutting behavior: This is a track path with strong cutting to the road center line within the area of the circular arc(conscious driving process to balance centrifugal acceleration).

(iv)Drifting behavior: This is asymmetrical track path between the beginning and end of the curve with a pronounced tendency to drive on the down direction at the beginning of the curve and an increasing drift to the up direction the end of the curve at left-hand curves; analogous at right-hand curves.
(v)Swinging behavior: This is asymmetrical track path between the beginning and end of the curve with a pronounced tendency to drive on the up direction at the beginning of the curve and an increasing drift to the down direction the end of the curve at left-hand curves; analogous at right-hand curves.

(vi)Correcting behavior: This is a track path with increased drifting toward the inside/outside of the curve and subsequent correction of the steering angle in the second half of the curve. It is assumed that this track type is a kind of unconscious driving behavior, due to underestimation of the curvature and/or the length of the curve.

Track paths that could not be assigned to those defined types are summarized in a separate group called "remaining" track paths.

The basic paths of the track types described above are illustrated in Fig. 1 by the example of a up direction. The sketches show the main points of the respective curves as well as the limits of the curve road cross sections $\mathrm{ZH}$ 
to HZ. And Table 1 gives a distribution probability of the track types per measurement direction in the curves investigated.

\subsection{Contrast analysis on track paths deviation characteristic of different direction}

Generally, the drivers operate their vehicles at a clearly larger distance from the road edge than from the centerline both in up direction and in down direction ${ }^{14-}$ 17. Tracks that represent the paths of the vehicle left front wheel are also different in different driving directions. The track types of up direction are obviously more than it of down direction. There may have following influencing factors: the lane widths, the sight distance, and the obstacles in roadside.

The averaged lapses of vehicle left front wheel along the three curved section in different directions can be analyzed as followed.

\subsubsection{Up direction}
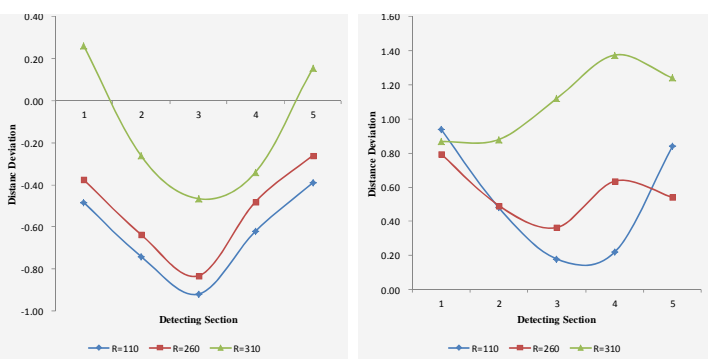

Normal（b)

Normal（ac）
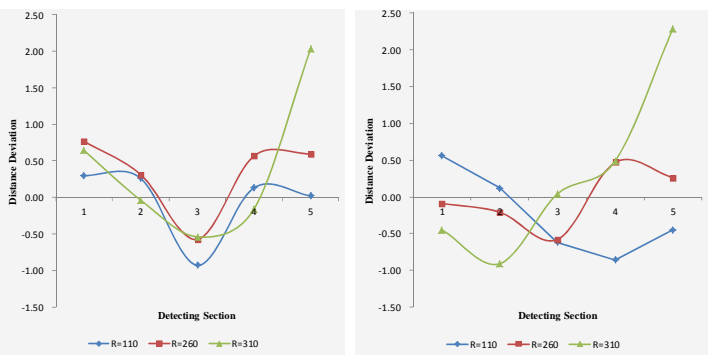

Cutting (de) Drifting (fghi)

Fig.2 contrast analysis of four main track types in up direction

The averaged lapses of vehicle left front wheel along the three curved section in stretched form in up direction can be shown in Fig.2. In up direction of curved section, four main track types were analyzed comparatively.
In Normal (b) behavior, the vehicle track paths cut the road center line more with the decrease of the radius, that is to say the track lapses increase with the decrease of the radius.

In Normal (a) and (c), the track lapses decrease with the decrease of the radius, that is to say the track paths nearer along to the road center line with the decrease of the radius.

In Cutting (d) and (e), the cutting value increase with the decrease of the radius, that is to say, with the decrease of radius, the track paths deviate more serious from the road center line in the road-cross section QZ. In Drifting (f) to (i), the track lapses turn into the curved section decrease, and the track lapses turn off the curved section increase with the decrease of the radius.

\subsubsection{Down direction}
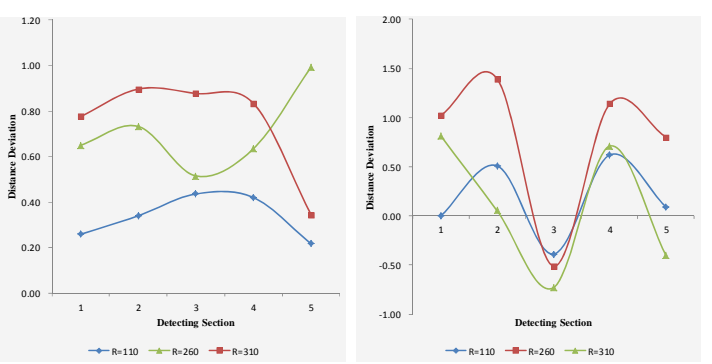

Normal（ac）
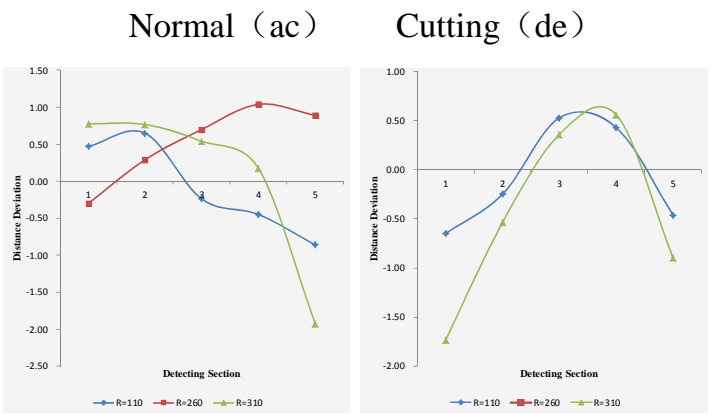

Drifting (fghi)

Correcting ( jk)

Fig.3 contrast analysis of four main track types in down direction

The averaged lapses of vehicle left front wheel along the three curved sections in stretched form in down direction can be shown in Fig.3. In down direction of curved section, four main track types were analyzed comparatively.

In Normal (a) and (c), the track lapses decrease with the decrease of the radius, that is to say the track paths 
nearer along to the road center line with the decrease of the radius.

In Cutting (d) and (e), the cutting value decrease with the decrease of the radius, that is to say, with the decrease of radius, the track paths deviate more slightly from the road center line in the road-cross section QZ.

In Drifting (f) to (i), the track lapses turn off the curved section increase with the decrease of the radius, but there is no obvious rules in the section of turn in.

In Correcting $(\mathrm{j})$ and $(\mathrm{k})$, the track lapse decrease with the decrease of the radius

\section{Probability of traffic accident in curved section based on vehicle track characteristic}

The analysis above shows that, opposite direction track crossing area will be created by up and down directions vehicle track when turn into or turn off the sharp curved section. Thus, the accident opportunity is offered by track crossing area to vehicles when they drive through the curved section. Because vehicle track paths data are obviously random, therefore, the characteristic of vehicle track crossing can be described by probability.

\subsection{The block division of curved road-cross section}

To transform vehicle track data into probability data, in this paper, the curved road-cross section was divided into different blocks firstly.

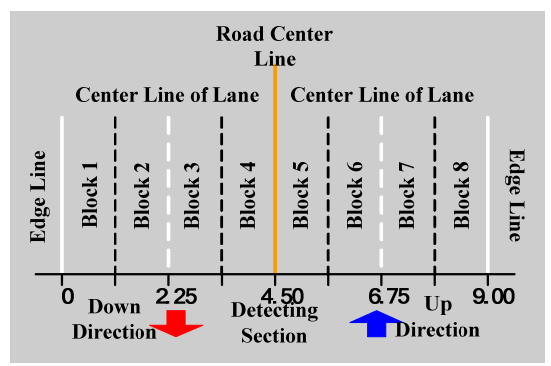

Fig.4 Sketches of Blocks

Taking into account the track data in this study is the distance from vehicle track point of left wheel to road inside edge line, the curved line which parallel with road inside edge line was chosen to divide the roadcross section. And at last, the road-cross section was divided into eight equal width blocks, which was defined as Block1 to Block8 from the road inside edge line. The sketch of blocks is can be seen as Fig.4.

\subsection{Probability of two vehicles' meets with in the curved section}

Three dispersed distribution law are used to describe the law of vehicle arriving, which is respectively the Poisson distribution (which is fit to small traffic flow density under the random condition), the Binomial distribution (which is fit to the comparatively congested traffic flow density under the condition of limited free driving opportunity) and the Negative Binomial distribution (which is fit to large wave properties of traffic flow density) ${ }^{18}$.

For this paper is mainly in the view of two-lane roads of secondary level or below, therefore, according to the $<$ Road Technical Standard $>$, convert various vehicles into small buses, two-lane roads of secondary level should adapt annual average car traffic volume of 500015000 one day. Thus, the traffic flow density isn't large and interactions among vehicles are weak, so the Poisson distribution can be used to describe the law of two-way two-lane road vehicle arriving.

As a result, probability of vehicles' arrives at some curved section can be calculated as the following formula:

$$
P(x)=\frac{(k s)^{x} e^{-k s}}{x !}, \quad x=0,1,2, \cdots, n
$$

In formula: $P(x)$ : Probability of vehicles' arrive at some same curved section; $k$ : vehicles space density, namely number of vehicles on the road with unit length in an instant in time; $s$ : length of road section; $e$ : the base of natural logarithms, take as 2,71828.

In a curved section of a certain length, during a period of time, $k_{t}$ : the average of spot density in the same section at all instants can be expressed as the following formula 4-2. The relationship among the section traffic flow volume, the average spot density and the average instantaneous velocity in the same road-cross section during a period can be well expressed by the following formula.

$$
\overline{k_{t}}=Q / 24 * \overline{u_{t}}
$$

In formula: $Q$ : the annual average daily traffic volume after converting various vehicles into passenger cars in a 
certain section; $u_{t}$ : the average instantaneous velocity of a certain section.

When the average instantaneous velocity is $70 \mathrm{~km} / \mathrm{h}$, the value of $k_{t}$ can be taken from Table.2.

In this paper, the value of $s$ of the test curved section is $2 \mathrm{~km}$, which include two sides straight section, and the value of $Q$ is 5000 pieces, average vehicle spot instantaneous velocity is $70 \mathrm{~km} / \mathrm{h}$, then the vehicle density of this curved section is 3pieces/lane under the condition of single lane, take stopping sight distance as $105 \mathrm{~m}$, then the probability of two vehicles' meets in this curved section is $P(x)=0.0181$.

\subsection{Probability of vehicle tracks cross in the same section but among different blocks.}

It can be known from above analysis that, the higher the probability of vehicle track crossing of different driving directions is, the higher the probability of vehicle accident is. Therefore, the vehicle accident probability from different driving direction can be defined as the vehicle track crossing probability, which is defined as followed.

Probability of tracks crossing: refer to the probability of vehicle tracks paths from different driving directions meet in a certain road-cross section. And in order to support driving safety of vehicles by the greatest extent, in this paper ,it assume that when vehicle tracks from different driving directions (up and down tracks) move into the same block, the up and down vehicle tracks are cross, namely track crossing.

Since vehicle tracks would fall into the different road block in different road-cross section, as a result, this paper define matrix $P_{s}(i, j)$ and $P_{x}(i, j)$ in order to express the distribution probability of up and down vehicle tracks in different road blocks respectively. In which, $i$ represents the five different observation roadcross sections of curved section, and $j$ represents the eight different equal width blocks of curved section. Then the probability of vehicle tracks crossing in a certain road-cross section among different blocks of curved section can be expressed by the following formula.

$$
P_{i}=\sum_{j=1}^{8} P_{s}(i, j) P_{x}(i, j) i=1,2, \cdots, 5
$$

According to the investigation, the distribution probability of vehicle track from different driving direction in different road blocks can be given in the Fig.5 and Fig.6

Then probability of vehicles accidents from different driving directions in each section is: $P_{1}=0.2254$; $P_{2}=0.4633 ; P_{3}=0.3834 ; P_{4}=0.4152 ; P_{5}=0.2751$

\subsection{Probability of traffic accident in curved section}

According to the analysis above, the probability of traffic accident in curved section can be expressed by the following formula.

$$
P_{a}=\sum_{i=1}^{n} P(x) P_{i} / n
$$

Probability of traffic accident in different sections of curved section is:

(i) Probability of traffic accident in different road-cross sections of curved section

Probability of traffic accident in observation section 1 $(\mathrm{ZH})$ is: $P_{a 1}=0.0041$;

Probability of traffic accident in observation section 2 (HY) is: $P_{a 2}=0.0084$;

Probability of traffic accident in observation section 3 (QZ) is: $P_{a 3}=0.0069$;

Probability of traffic accident in observation section 4 $(\mathrm{YH})$ is: $P_{a 4}=0.0075$;

Probability of traffic accident in observation section 5 (HZ) is: $P_{a 5}=0.0050$.

(ii) Probability of traffic accident in the whole curved section

$$
P_{a}=\sum_{i=1}^{n} P(x) P_{i} / n=0.064
$$

\section{Driving Dangerous Area Division and Feature Analysis Based on the Vehicle Track Paths}

According to the above analysis on the change of vehicle track paths in curved section, the drivers will successively run through three dangerous areas when driving on curved sections:

\subsection{Driving Dangerous Area I}

Inward Deviation Area of Track paths: the spatiotemporal area with larger objective risk and poor subjective sense of security.

It would be hard for drivers to understand the objective road information in the front when they just turn into the 
curved section. Drivers are in the process of wait and see at this time. Vehicle track deviation would be conducted as long as drives consider there is no traffic conflict risk in the front. This dangerous area is located at the section turn into the curved section, and shows a long belt type distribution in zones. It shows the highest risk in terms of vehicle track paths features and the calculation result of accident probability.

\subsection{Driving Dangerous Area II}

Track Crossing Area: the spatiotemporal area with maximum objective risk and better subjective sense of security.

In order to adapt the higher speed, vehicle track lapses from the road center line often be conducted by drivers to balance the centrifugal acceleration. Under the influence of various factors, such as the strongly reduced sight distance, the relatively higher speed and so on, driver always are confront with the higher traffic conflict risk from the opposite driving direction. This dangerous area is located at the middle of the curved section, and shows a short belt type distribution in zones. And the vehicle from up direction will meet the higher risk compare with down direction. It shows the higher risk in terms of vehicle track paths features and the calculation result of accident probability.

\subsection{Driving Dangerous Area III}

Outward Deviation Area of Track paths: the spatiotemporal area with minimum objective risk and best subjective sense of security.

Drivers have a basic understanding of the objective road information in the front when they driving past the middle of curved section. At this time, there usually are two behavior conducted by drivers, one is driving in the opposite direction near the road center line still, and the other is back to the original lane. This dangerous area is located at the section turn off the middle of the curved section, and shows a point type distribution in zones. It shows the lower risk in terms of vehicle track paths features and the calculation result of accident probability.

From the driving decision making process, the formation of vehicle track paths is a changing process continuously, that is to say, the latter track path is influenced by the former. And for the driving dangerous area, its spatiotemporal area also is influenced by the driving behavior decision making from the former driving dangerous area, except the factors such as the curve radius, the driving sight distance and so on.

\section{Conclusion}

The types, deviation and crossing characteristics of vehicle track are well studied in this paper, which reflect the deviation of driving expectation from real road traffic condition, and also related to the driving stability and security.

One promising result in this paper is that the accident probability based on the vehicle track in each observation section can be calculated, and the distribution probability of each track types is also calculated. Thus, there are three driving dangerous areas in terms of the vehicle track deviation and track crossing, and the feature and the accident risk is different from each other.

But for the complexity of traffic behavior, this paper only studied the influence from deviation characteristic of track without the influence of other vehicles in the same driving direction or in the opposite driving direction. Therefore, the result in this paper only reveals partly relationship between traffic behavior and driving security. Vehicle track behavior is influenced by others and also vehicle speed behavior both should be combined to analyze driving security comprehensively in the following study.

Table.2 Value-taking reference form of $\bar{k}_{t}$ under $\bar{u}_{t}$ is $70 \mathrm{~km} / \mathrm{h}$

\begin{tabular}{cc|c|c|c|c|c}
\hline Density & Traffic volume & 5000 & 7000 & 9000 & 11000 & 15000 \\
\hline $\bar{k}_{t}$ & 2.98 & 4.17 & 5.36 & 6.55 & 8.93 \\
\hline
\end{tabular}




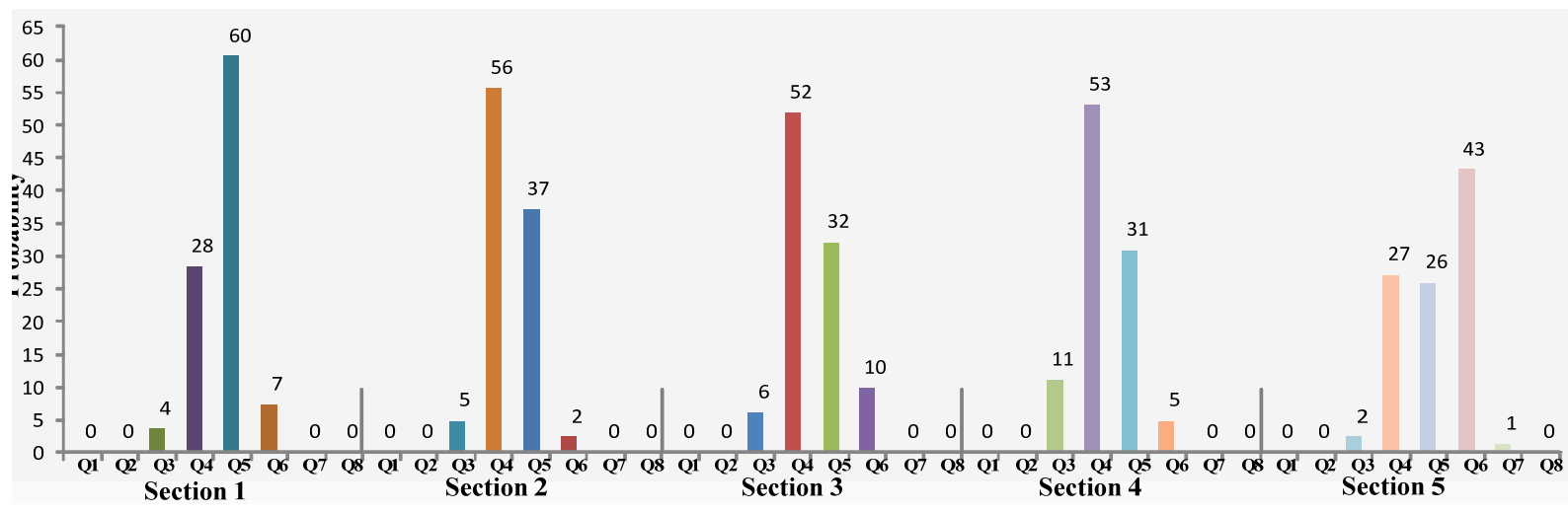

Fig.5 Distribution frequency of vehicle track of up direction in different road blocks

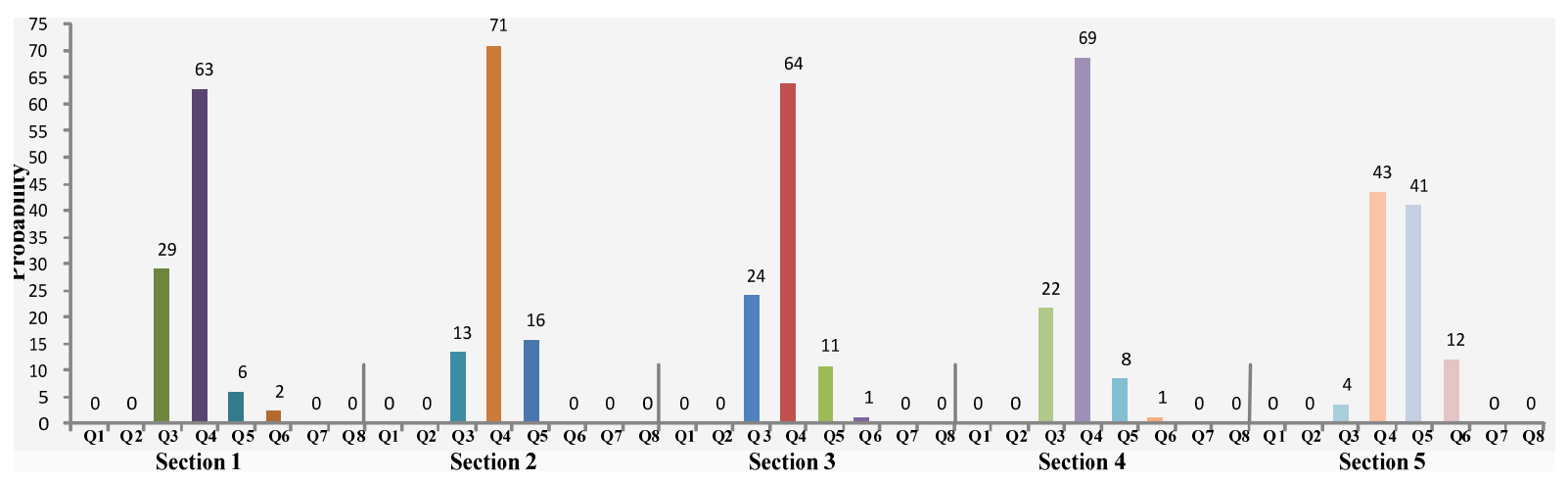

Fig.6 Distribution frequency of vehicle track of down direction in different road blocks

\section{Acknowledgements}

This work was supported by National Natural Science Foundation of China (No.50978114) and Project 20101016 Supported by Graduate Innovation Fund of Jilin University.

\section{References}

1. J. D. Lee, Fifty Years of Driving Safety Research, Human Factors and Ergonomics Society, 50(3) (2008), 521-528.

2. G. Zhou, Analysis of Influencing Factors on Freeway Traffic Safety and Fuzzy Comprehensive Evaluation, M. S. Thesis (in Chinese), Southwest Jiaotong University, (2004)

3. Y. Y. Wang, Research on the Accident Causations of Mountainous Road, M. S. Thesis (in Chinese), Beijing University of Technology, (2008)

4. I. L. Thomas and W. T. Taylor, Effect of Edge Striping on Traffic Operations, Highway Research Board Bulletin, 244(1960), 11-15.
5. X. D. Sun, Impact of Edge Lines on Safety of Rural TwoLane Highways. Civil Engineering Department University of Louisiana at Lafayette, (2005)

6. P. Spacek, Track Behavior and Accident Occurrence in Curves on Two-lane Highways in Rural Areas. 2nd International Symposium on Highway Geometric Design, (2000), 288-298

7. P. Spacek, Track Behavior in Curve Areas: Attempt at Typology, Journal of Transportation Engineering, 131(9) (2005), 669-677

8. E. Donges, A two level model of driver steering behavior, Human Factors, 20(6) (1978), 691-707.

9. A. Garcia and E. Diaz, Vehicle Tracking in Black Spots Using Artificial Vision Techniques in Order to Improve Road Geometric Design Standards, International Symposium on Highway Geometric Design Practices, (EC003) (1998), 38:1-6

10. H. Feng, Research on the Establishment of Traffic Signs and Marking in Two-lane Mountain Highway, M. S. Thesis (in Chinese), Jilin University, (2007)

11. R. Krammes, R. Brackett, et al, Horizontal Alignment Design Consistency for Rural Two-Lane Highways, 
Federal Highway Administration Report, (Washington, D. C. 1995)

12. M. Islam and P. Seneviratne, Evaluation of Design Consistency of Two-Lane Rural Highways, ITE Journal, 64(02) (1994), 28-31

13. J. C. Glennon and G. D. Weaver, The Relationship of Vehicle Paths to Highway Curve Design. (Texas Transportation Institute, 1971)

14. B. Liu, Mechanism Study on the Vision-based Speed Control and lane Keeping, M. S. Thesis (in Chinese), Wuhan University of Technology, (2008)

15. J. Xu, Effects of Change in Road Geometry Design on Vehicle Driving Dynamics, M. S. Thesis (in Chinese), South Jiaotong University, (2010)

16. T. Lin, Vehicle Acceleration and Driving Track Research of Sharp Curves in Mountainous Highway, M. S. Thesis (in Chinese), Tongji University, (2008)

17. Z. Yang, Research on Driving Dynamic Simulation Model, Ph. D. dissertation (in Chinese), Tongji University, (2004)

18. D. H. Wang, Traffic Flow Theory, (China Communications Press, China, 2002)

19. Y. Y. Ren, X. S. Li, Study on distribution characteristics of dangerous driving area in curved section based on human-vehicle operation characteristics, 2010 IEEE International Conference on Emergency Management and Management Sciences (ICEMMS), (2010), 189-193

20. S. Upadhyaya, A. Arora and R. Jain, Reduct Driven Pattern Extraction from Clusters, International Journal of Computational Intelligence Systems(IJCIS), 2(1) (2009), $10-16$

21. A. Assenza, M. Valle, M. Verleysen, A Comparative Study of Various Probability Density Estimation Methods for Data Analysis, International Journal of Computational Intelligence Systems(IJCIS), 1(2) (2008), 188-201

22. Wuhong Wang,Wei Zhang,Hongwei Guo,Heiner Bubb,Katsushi Ikeuchi, A safety-based behavioural approaching model with various driving characteristics, Transportation Research Part C-Emerging Technologies, doi:10.1016/j.trc.2011.02.002.

23. Wuhong Wang, Qi Cao, Katsushi Ikeuchi, Heiner Bubb, Reliability and safety analysis methodology for identification of drivers' erroneous actions, International Journal of Automotive Technology, 2010 11(6),873-881.

24. Wuhong Wang, Yan Mao, Jin Jing, Xiao Wang, Hongwei Guo, Xuemei Ren, Ikeuchi Katsushi, Driver's various information process and multi-ruled decision-making mechanism: a fundamental of intelligent driving shaping model, International Journal of Computational Intelligence Systems,2011 4(3),297-305. 